\title{
Therapeutic Guidelines: Ulcers and wound management. Version 2
}

\section{Kanthi Vemuri \\ Infectious diseases advanced trainee, Sunshine Coast University Hospital, Queensland}

\section{Aust Prescr 2019;206}

https://doi.org/10.18773/ austprescr.2019.064

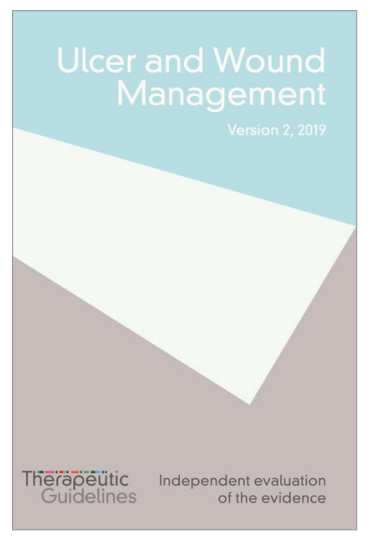

Melbourne: Therapeutic Guidelines Limited; 2019. 155 pages

Also available at www.tg.org.au

This latest edition is a brilliant, informative guide to managing ulcers and wounds. It provides a succinct summary and a practical, easy-to-follow framework for managing these challenging conditions. The tables, boxes, figures and illustrative photos help to easily navigate through the important facts, in a timely fashion.

I think the best aspect of this book is that it highlights the importance of early establishment of an aetiology of the ulcer in tailoring the management. This edition has a chapter on skin tears which are so common in older people. It provides information on prevention, assessment and how to avoid complications such as infection and conversion into a chronic ulcer. The chapter on wounds in the high-risk foot describes the importance of prevention and the need to focus on revascularisation and pressure-redistribution strategies during the early assessment.

The emphasis on non-pharmacological measures for ulcer healing such as foot care, foot wear, compression stockings and appropriate dressings highlights the vast experience the expert group has in this field. The well-designed tables provide an excellent guide to choose the appropriate dressings depending on wound characteristics. There is also a new section about a palliative approach to ulcers that are not expected to heal, including pain management and dressing choice.

I highly recommend this latest edition, which incorporates all the known successful strategies, to every clinician involved in ulcer and wound management, both in the hospital and community. 